\title{
Probabilistic Constellation Extension Scheme for PAPR Reduction in OFDM Signals
}

\author{
Manish K. Patidar ${ }^{* a}$, A. Mishra ${ }^{a}$ and R. Saxena ${ }^{b}$ \\ ${ }^{a}$ Department of Electronics and Communication Engineering, Jaypee University of Engineering and \\ Technology, A. B. Road Raghogarh-473226 District-Guna, Madhya Pradesh, India. \\ ${ }^{b}$ Director, Jaypee University Aligarh Road, Anoopshahr-203390 District-Bulandshahr, Uttar Pradesh, India.
}

\begin{abstract}
In this paper, a combinational method is proposed to mitigate the peak-to-average power ratio problem in orthogonal frequency division multiplexing signals. The proposed algorithm is an intelligent combination of the constellation extension and probabilistic techniques. It is shown that proposed technique achieves the significant reduction in peak-to-average power on maintaining sufficient bit error rate with a slight increase in computational complexity compared to conventional schemes.
\end{abstract}

Index Terms: Active Constellation Extension (ACE), Approximate Gradient Project (AGP), Partial Transmit Sequence (PTS), Project onto convex set (POCS) and Selective Mapping (SLM).

(C) 2015 Published by MECS Publisher. Selection and/or peer review under responsibility of the Research Association of Modern Education and Computer Science

\section{Introduction}

The growing demands of wireless communication require large data rate transmission with high mobility. Multi carrier modulation (MCM) fulfils this requirement very effectively and works very robust in fading channel as well as interference. One of the important MCM technologies is orthogonal frequency division Multiplexing (OFDM) which applied in several applications such as IEEE 802.11a, IEEE 802.16, Digital Audio Broadcasting (DAB), Digital Video Broadcasting (DVB), HIPERLAN/2 and 4G mobile communication systems. There are some issues still remain challenging in OFDM system like high peak-to average power ratio (PAPR), timing and frequency synchronization and phase offset. The PAPR problem is mostly associated with uplink since the efficiency of radio frequency (RF) power amplifier is critical due to the limited battery power in a mobile terminal.

Recently, various methods have been proposed to reduce the PAPR [1-10]. These methods includes coding [2], clipping [3], tone reservation (TR), tone injection (TI) [4], selective mapping (SLM) [5, 6], partial transmit

* Corresponding author. Tel.: +919424055046

E-mail address: er.manipatidar@gmail.com 
sequence (PTS) [7, 8] and active constellation extension (ACE) [9, 10]. The comparison among all above techniques [1] shows that probabilistic approaches posses lesser PAPR, i. e. SLM, PTS, TR and TI. On the other hand, ACE gives constellation in non bijective way in which points extends to minimize peak power of signal. This can be done by two algorithms i.e. project onto convex set (POCS) [11] and approximate gradient project (AGP) [10].

The objective of this paper is in the direction of combining of two techniques i.e. AGP and PTS, in such a way that PAPR is reduced and bit error rate (BER) is maintained as compared with other conventional techniques. In Section II, OFDM model and PAPR is formulated. PAPR reduction schemes describes in section III. Proposed PAPR combinational algorithm and computational complexity are discussed in section III. Finally results and conclusion are presented in section IV and V respectively.

\section{OFDM System}

An OFDM signal is represented in the form of $\mathrm{N}$ independent subcarriers which are orthogonal to each other with frequency separation of $1 / T$, where, $T$ is the orthogonal symbol period. However, these orthogonal frequencies are generated with the help of Discrete Fourier Transform (DFT). The transmitted envelop of OFDM can be expressed as-

$$
y[n]=\frac{1}{\sqrt{N}} \sum_{k=0}^{N-1} Y_{k} e^{j 2 \pi k n / N} \quad 0 \leq n \leq N-1
$$

Here, information is transmitted in the form of constellation and depends upon type of mapping used such as BPSK, QPSK and QAM. After computing inverse Discrete Fourier Transform (IDFT), when all subcarriers are added with the same phase, they produce a peak power which is $N$ times the average power. The envelope fluctuation creates high values of some peak signal in spectrum to corresponding average values. This increase in peak-to-average power ratio (PAPR) [1] of OFDM and represented as-

$$
P A P R[y[n]]=\frac{\max _{0 \leq n \leq N-1}\left[|y[n]|^{2}\right]}{E\left[|y(n)|^{2}\right]}
$$

where, E[.] in (2) denotes the expectation operator. The measurement of PAPR is done in terms of complementary cumulative distribution function (CCDF).

\section{PAPR Reduction Techniques}

In this section, probabilistic and constellation extension approaches are discussed. In both approaches two important methods are presented.

\subsection{Probabilistic}

The term probabilistic is also known as scrambling in multicarrier communication. In this approach input data is scrambled and multiplied with suitable phases so that a minimum PAPR sequence is obtained. The main examples of probabilistic approach are SLM and PTS methods.

\subsubsection{Selective Mapping}

Selective mapping, proposed by van Eetvelt et al. [5] and B"auml et al. [6], creates partition in the sequence which are then multiplied by different phase array and sequence with minimum peak to average power chosen 
from all block. The OFDM sequence $Y[K]=[Y[0],[1], \ldots, Y[N-1]]$ is divided into U block having same size as $Y[K]$.

All these separate blocks are multiplied by phase sequence $G^{U}=\left[G_{0}^{U}, G_{1}^{U}, \ldots, G_{N-1}^{U}\right]^{T}$, have same length as the original OFDM sequence. This produce new modified sequence i.e. $Y[K]^{U}=\left[\left\{Y^{U}[0], Y^{U}[1], \ldots, Y^{U}[N-\right.\right.$ $1]]^{T}$. The IDFT of all block sequences are computed as-

$$
y^{U}[n]=\left[y^{U}[0], y^{U}[1], \ldots, y^{U}[N-1]\right]^{T}
$$

Among which, the one, with the lowest peak power is chosen from (3) for transmission i.e. $\bar{y}=y^{U}$. The selective phase sequences are transmitted to receiver for recovering the original ODFM sequence, known as side information [12].

$$
\breve{U}=\operatorname{argmin}_{u=1,2, ., U}\left(\max _{n=0,1, \ldots, N-1}\left|y^{u}[n]\right|\right)
$$

\subsubsection{Partial Transmit Sequence}

The PTS method, proposed by M"uller and Huber [7] and Tellambura [8] presents an efficient method for phase factor computation. The OFDM mapped sequence partitions into $\mathrm{U}$ disjoint sub blocks $Y[K]=$ $\left[Y^{0}, Y^{1}, \ldots Y^{U-1}\right]^{T}$, which are consecutively located. The sub blocks of equal in size with zero padding at vacant position, known as partial sequence, are transformed into time domain and collected the phase information from respective sub block. With the help of phases of partial sequence, the new complex phase vectors $g^{u}=e^{j \varphi u}, u=1,2, \ldots, U$ are generated. These partial sequences are multiplied to corresponding phases. The phase vector is chosen so that the PAPR can be minimized, which is shown as -

$$
\bar{y}[n]=\sum_{u=1}^{U} g^{u} y^{u}
$$

Then, the time-domain signal with the lowest PAPR, shown in equation (5) is transmitted along with side information of phases to recover the original OFDM sequence.

$$
\left[\check{\mathrm{p}}^{1}, \ldots, \check{\mathrm{p}}^{\mathrm{U}}\right]=\operatorname{argmin}_{\left[\mathrm{p}^{1}, \ldots \mathrm{p}^{\mathrm{U}}\right]}\left(\max _{\mathrm{n}=0,1, \ldots, \mathrm{N}-1}\left|\sum_{\mathrm{v}=1}^{\mathrm{U}} \mathrm{p}^{\mathrm{u}} \mathrm{y}^{\mathrm{u}}[\mathrm{n}]\right|\right)
$$

In general, the selection of the phase vectors in probabilistic approach is very difficult. Therefore, the search complexity increases with sub blocks. The main difference between the SLM and PTS is scrambling, which applied to all subcarriers and each sub block respectively.

\subsection{Active Constellation Extension}

The ACE, proposed by Krongold and Jones [10], is a convex problem whose solution is guaranteed to be a global minimum PAR solution given with ACE constraints [11]. 


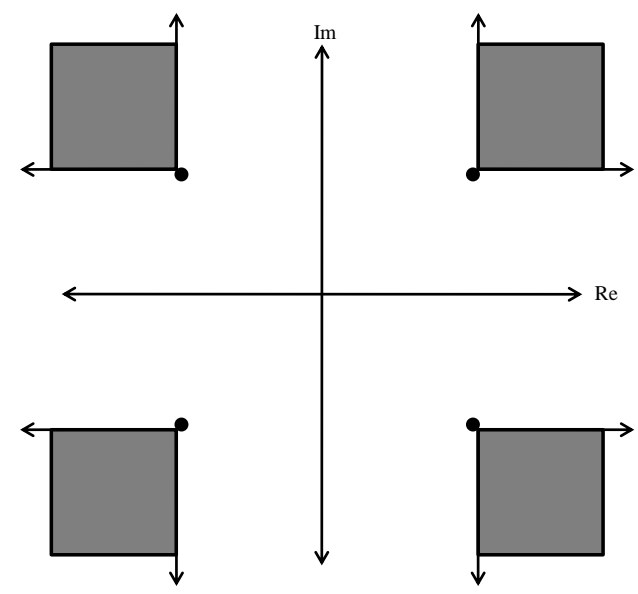

Fig. 1. Constellation Extension for QPSK in ACE schemes

It requires both time-domain and frequency-domain signal processing. The main principle of this technique is to shift the outer constellation points towards exterior of original constellation generating an alternative representation of the same symbol. Fig. 1 shows the corresponding extension of each point for QPSK. In a literature $[9,10]$, two variance of ACE algorithms are presented i.e. projection onto convex sets (POCS) and approximate gradient project (AGP).

\subsubsection{Project onto Convex Sets}

POCS have two convex sets that determine the extended constellation and interior points. The algorithm perform clipping at predefined level $C$ but the phase remains same as modulated sequence-

$$
\bar{y}[n]=\left\{\begin{array}{ll}
y[n] & |y[n]|<M \\
M e^{j \theta[n]} & |y[n]| \geq M
\end{array}\right\}
$$

where, $y[n]=|y[n]| e^{j \varphi[n]}$

The $\bar{y}[n]$ from (5) is transformed into $Y[K]$, enforced all constellation extension constraints by restoring all interior points to their original values, while projecting points into the increased margin. This process continues until clipping is zero or the PAPR is minimized. The POCS scheme suffers the problem of slow convergence and also difficulty to choose appropriate clipping level.

\subsubsection{Approximate Gradient Project}

Steepest gradient direction is used in AGP algorithm to minimize the peak value. This algorithm is formulated by considering the clipped signal $m_{\text {clip }}$, which is expressed as -

$$
\bar{y}[n]=y^{c}[n]+m_{\text {clip }}[n]
$$

where , 
$m_{\text {clip }}[n]=\left\{\begin{array}{cc}0, & |y[n]| \leq M \\ \left(M-\left|y^{c}[n]\right|\right) e^{j \theta[n]} & |y[n]|>M\end{array}\right.$

The importance of this algorithm is ACE constraints [11] for extended constellation as illustrated in Fig. 1. Finally, new OFDM sequence can be obtained as-

$$
y^{c+1}=y^{c}+\delta m_{\text {ext }}
$$

where, $c$ is number of iteration,$\delta$ is gradient and $m_{\text {ext }}$ is extended time sequence in (10). This process end with acceptable PAR or a maximum iteration count has reached.

\section{Proposed Technique}

The PAPR techniques require improvement for other parameters such as BER, computational complexity and so on [1, 11]. Fig.2 shows different combinational technique for PAPR reduction. The AGP-PTS produces less PAPR compare to other techniques, because of their individual performance are better than other methods.

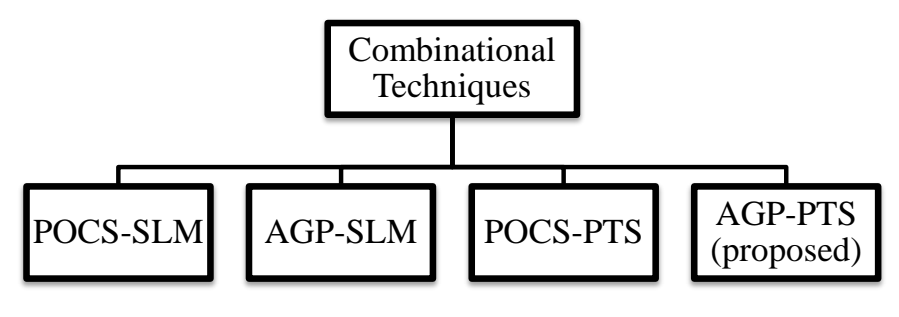

Fig.2. Various combinational techniques for PAPR reduction

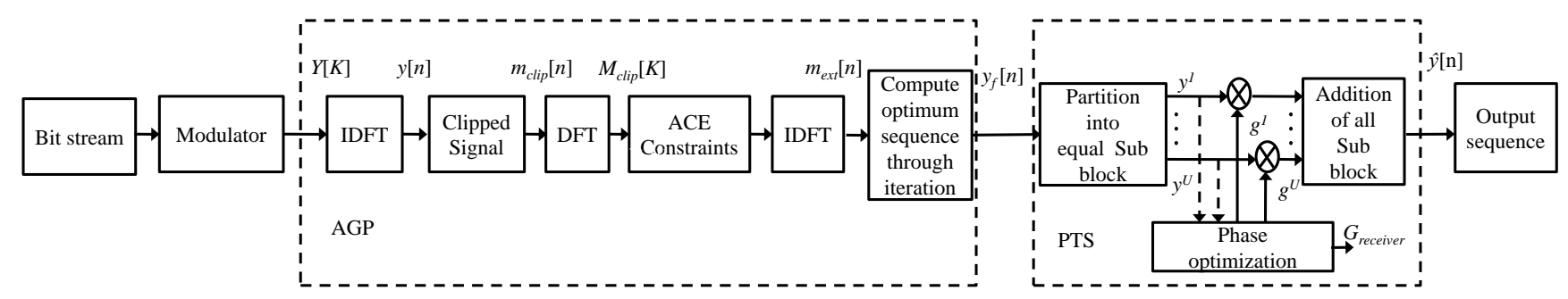

Fig. 3. Block diagram of proposed scheme

The algorithms for proposed technique shown in Fig. 3 is as follows-

(1) Get an OFDM symbol in frequency-domain $Y[K]$ from modulator.

(2) Convert this symbol into time-domain $\mathrm{y}[n]$ using IDFT.

(3) Perform AGP on $y[n]$. Initialize with iteration, $c=0$ and set maximum value to $c_{\max }$.

(4) Apply clipping level $M$ on $y[n]$ and preserve same phase as in original OFDM symbol-

$$
\bar{y}[n]=\left\{\begin{array}{ll}
y^{c}[n] & \left|y^{c}[n]\right|<M \\
M e^{j \varphi[n]} & \left|y^{c}[n]\right| \geq M
\end{array}\right\}
$$


where, $y^{i}[n]=\left|y^{c}[n]\right| e^{j \varphi[n]}$

(5) Obtain clipped signal portion of equation (11)-

$m_{\text {clip }}[n]=\bar{y}[n]-y^{c}[n]$

(6) Transform $m_{\text {clip }}$ to frequency domain i.e. $M_{\text {clip }}$.

(7) Set direction of constellation point according to ACE constraints. It means restore all interior constellation point to original location and project exterior points to outer side of constellation as illustrated in Fig. 1.

(8) Apply IDFT to obtain new extended constellation i.e. $m_{\text {ext }}$.

(9) Select suitable gradient $\delta$ and compute new sequence by equation -

$y^{c+1}[n]=y^{c}[n]+\delta m_{\text {ext }}[n]$

(10) Perform check operation on $y^{c+1}[n]$. There are two conditions for checking. First, the PAPR comparison with original sequence and second, iteration has been reached to maximum.

(11) Update $c=c+1$ and get final value of $y_{f}[n]$.

(12) Now, apply PTS to $y_{f}[n]$. Partition the sequence into $U$ disjoint sub block of equal size.

(13) Generate set of phase vector $g=\left[g^{1}, g^{2}, \ldots, g^{U}\right]$ and multiply with partial sequence $y_{f}[n]=$ $\left[y^{1}, y^{2}, \ldots, y^{U}\right]$.

(14) Perform check operation equal to candidate sequence i.e. $M=4^{U-1}$ for minimum PAPR symbol to original signal.

$\hat{y}[n]=\sum_{u=1}^{U}\left[g^{u} * y^{u}[n]\right]$

(15) Consider minimized PAPR sequence in (14)

(16) $\left.G_{\text {reciver }}={ }_{\left[g^{1}, \cdots, g^{U}\right]}^{\arg }{ }_{n=0,1, \cdots, N-1}|\hat{y}[n]|\right)$

(17) Prepare side information for receiver to know the corresponding phase sequence by (15)

\section{Computational Complexity}

The complexity of proposed method is evaluated in terms of multiplications and addition. The PAPR reduction schemes have complex nature of computation. For a probabilistic approach computation of complexity is done with $\mathrm{N}$ (number of subcarrier), $\mathrm{U}$ (sub block) and $n$ (bit information i.e. $n=\log _{2} N$ ). The complexity of SLM and PTS in terms of number of complex multiplications per OFDM symbol is respectively, $U \times N \times[(n / 2)+1]$ and $U \times N \times n / 2$, while the number of complex additions is $U \times N \times n$ and $(M+4 \times U+2) \times N \times n$ respectively [11]. Here, $M$ is number of candidate sequence as shown in step 14. As $U$ increases PAPR reduces but complexity increases. On the other hand, constellation extension methods perform many iterations (i.e. $c$ ) to converge. Therefore, POCS requires $c \times N \times n / 2$ complex multiplications and $c \times N \times n$ complex additions, while AGP needs complex multiplications $c \times\{2 \times N+(N \times n) / 2\}$ and complex additions $c \times\{4 \times N+N \times n\}$. However, computational complexity for series cascade combination technique can be calculated through addition of both techniques on removing common IFFT.

\section{Results}

This section provides simulation results along with discussion on the performance of conventional and proposed techniques. In simulation, 10,000 QPSK modulated OFDM symbol for IEEE 802.11a and IEEE 
802.16e standard subcarrier. Simulation set up for SLM and PTS divides sequence into four sub blocks, followed by POCS and AGP perform with two iterations.

In Fig.4 (a) it is shown that proposed method reduces PAPR by $0.4 \mathrm{~dB}$ as compared to PTS method and 0.2 $\mathrm{dB}$ to POCS-PTS at $0.0024 \mathrm{CCDF}$ [13]. This schemes further reduces the PAPR by increasing the subcarriers $(\mathrm{N}=256)$. It is also shown in Fig. 4 (b) the improvement of $2 \mathrm{~dB}$ is found to conventional methods i.e. AGP and PTS both on 0.0027 CCDF. Fig.5 (a) and (b) illustrates that in presence of additive white Gaussian noise (AWGN), the proposed schemes maintains the same BER. Moreover, for 256 subcarriers the BER performance is found better than conventional method at $8 \mathrm{~dB}$ signal-to noise ratio (SNR). The power spectral density graphs of proposed serial method for both the OFDM standards are shown in figure 6 (a) and b respectively. In proposed method, though the in-band-ripples have small ripples but out-of-band radiations are significantly lesser than the other schemes.

The computational complexity $[14,15]$ of conventional and proposed methods is summarized in Table 1 . Though POCS-SLM shows least complexity among combinational techniques but it posses high PAPR than proposed AGP-PTS technique. From Table 1, it is illustrated that in series cascade technique, complex multiplication is increased by huge margin i.e. $85 \%$ and $76 \%$ in WLAN and WiMAX standards respectively and at the same time, $4 \%$ increment in complex addition for both standards compared to PTS.

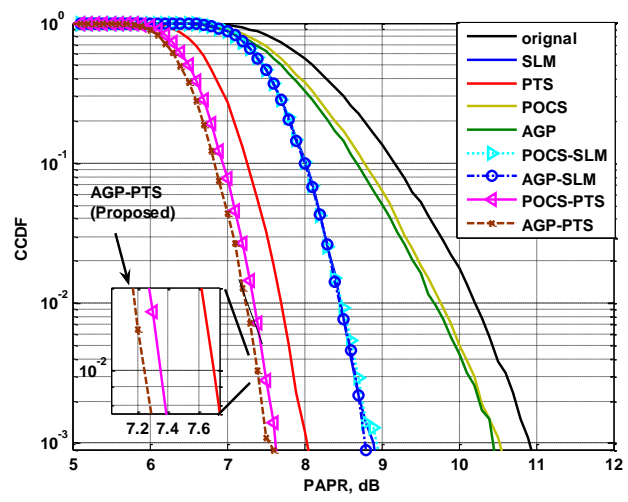

(a)

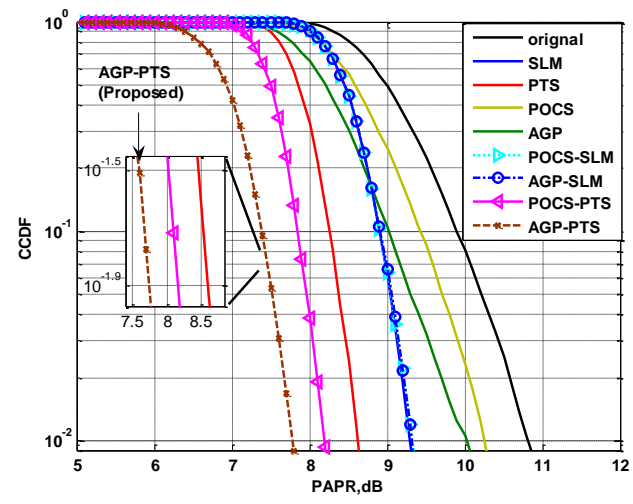

(b)

Fig. 4. PAPR comparison (a) IEEE 802.11a (b) IEEE 802.16e

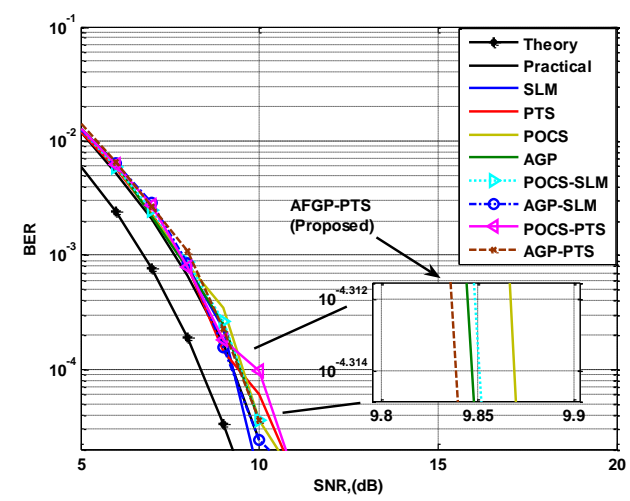

(a)

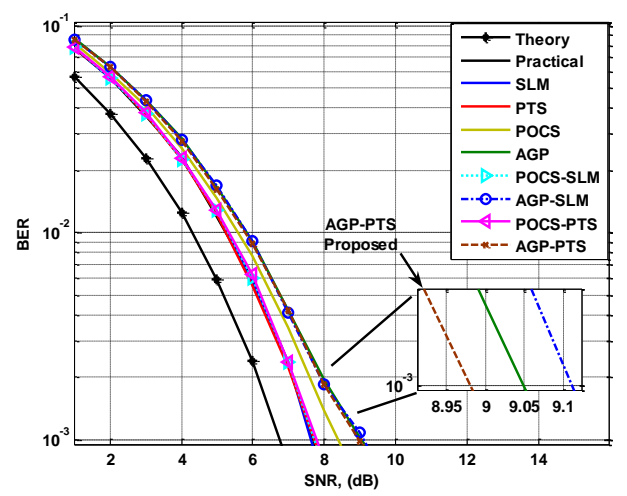

(b)

Fig. 5. BER performance (a) IEEE 802.11a (b) IEEE 802.16e 


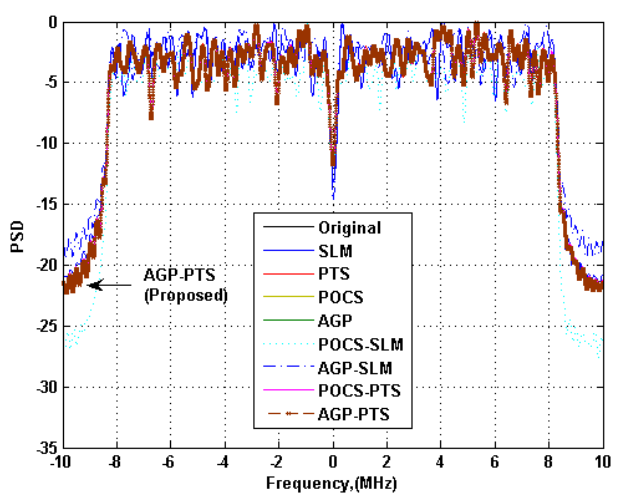

(a)

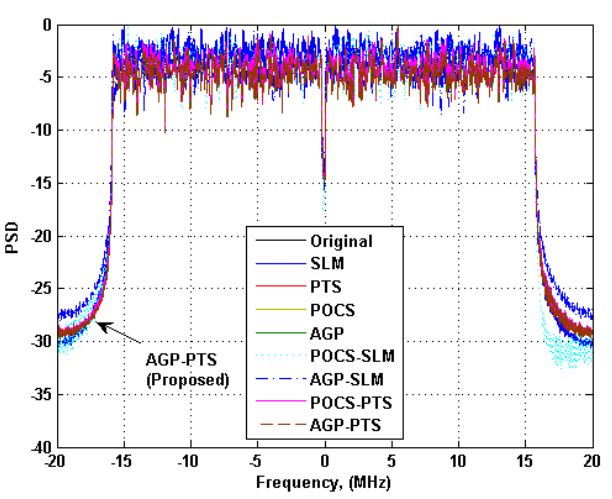

(b)

Fig. 6. Power spectrum performance (a) IEEE 802.11a (b) IEEE 802.16e

Table 1. Computational Complexity

\begin{tabular}{|c|c|c|c|c|c|c|c|c|}
\hline $\begin{array}{l}\text { Techniques } \\
\text { Parameters }\end{array}$ & SLM & PTS & POCS & AGP & POCS-SLM & AGP-SLM & POCS-PTS & $\begin{array}{c}\text { AGP-PTS } \\
\text { (proposed) }\end{array}$ \\
\hline \multicolumn{9}{|c|}{ IEEE 802.11a } \\
\hline $\begin{array}{c}\text { Complex } \\
\text { Multiplication } \\
\end{array}$ & 801 & 593 & 296 & 504 & 1097 & 1305 & 889 & 1097 \\
\hline Complex Addition & 1186 & 24307 & 593 & 1009 & 1779 & 2195 & 24900 & 25316 \\
\hline \multicolumn{9}{|c|}{ IEEE 802.16e } \\
\hline $\begin{array}{c}\text { Complex } \\
\text { Multiplication } \\
\end{array}$ & 3858 & 3058 & 1529 & 2329 & 5386 & 6186 & 4586 & 5386 \\
\hline Complex Addition & 6115 & 125359 & 3058 & 4658 & 9173 & 10773 & 128417 & 130017 \\
\hline
\end{tabular}

\section{Conclusion}

In this paper, various combinational techniques for PAPR reduction are presented. Out of which, the proposed scheme is a series combination of AGP and PTS method. The proposed scheme provides a good performance in PAPR reduction as well as in BER to conventional methods. Moreover, for large number of subcarrier, the PAPR further reduces and simultaneously BER improves. The proposed method reduces the PAPR significantly $(1 \mathrm{~dB})$, maintains the BER performance as well spectral spreading compared to conventional technique. However, the increment of $85 \%$ and $76 \%$ has been observed in complex multiplications for WLAN and WiMAX respectively.

\section{References}

[1] T. Jiang and Y. W. Wu, "An overview: Peak to average power ratio reduction techniques for OFDM signals," IEEE Trans. Broadcast., vol. 54, no.2, pp. 257-268, 2008.

[2] K. Patterson, "Generalized Reed-Muller codes and power control in OFDM modulation," IEEE Trans. Inf. Theory, vol. 46, no.6, pp. 104-120, 2000.

[3] X. Li and L.J. Cimini Jr, "Effects of clipping and filtering on the performance of OFDM," IEEE Commun. Lett., vol. 2, no.5, pp. 131-133, 1998. 
[4] J. Tellado, "Peak power reduction for multicarrier transmission," PhD thesis, University of Stanford, Stanford, 1999.

[5] P.W. J. van Eetvelt, G. Wade, and M. Tomlinson, "Peak to average power reduction for OFDM schemes by selective scrambling," Elect. Lett., pp. 1963-1964, 1996.

[6] R.W. B"auml, R. F. H. Fischer, and J. B. Huber, "Reducing the peak-to-average power ratio of multicarrier modulation by selected mapping," Elect. Lett., vol. 32, no. 22, pp 2056-2057, 1996.

[7] S. H. Muller and J. B. Huber, "OFDM with reduced peak-to-average power ratio by optimum combination of partial transmit sequences," Elect. Lett., vol. 33, no. 5, pp. 368-369, 1997.

[8] C. Tellambura, "Improved phase factor computation for the PAR reduction of an OFDM signal using PTS", IEEE Commun. Lett., pp. 135-137, 2001.

[9] B. S. Krongold and D. L. Jones, "PAR reduction in OFDM via active constellation extension," IEEE Trans. Broadcast., vol. 49, no. 3, pp. 258-268, 2003.

[10] D. L. Jones, "Peak power reduction in OFDM and DMT via active channel modification," Proc. Asilomar Conference on Signals, Systems, and Computers, vol. 2, pp. 1076-1079, 1999.

[11] D.-W. Lim, S.-J. Heo and J.-S. No, "An overview of Peak-to-Average Power Ratio Reduction Schemes for OFDM signals," Journal of commun. and networks, vol 11 ,no.3, pp 229-239, 2009.

[12] S. Raja, I. Hossain, K. H. Jewel, S. Al, "PAPR Reduction of OFDM Signal Using Sequential Phase Sequence SLM Based Transceiver without Side Information," International Journal of Wireless and Microwave Technologies, vol. 5, no. 1, pp. 34-42, 2015.

[13] S. N. Sur and D. Ghosh, "Channel Capacity and BER Performance Analysis of MIMO System with Linear Receiver in Nakagami Channel”, International Journal of Wireless and Microwave Technologies, vol. 3, no. 1, 2013.

[14] S. H. Han and J. H. Lee, "An overview of peak-to-average power ratio reduction techniques for multicarrier transmission," IEEE Wireless Communications, vol. 12, no. 2, pp. 56-65, 2005.

[15] Y. Rahmatallah and S. Mohan, "Peak-To-Average Power Ratio Reduction in OFDM Systems: A Survey and Taxonomy", IEEE Communications Surveys \& Tutorials, vol. 15, no. 4, pp. 1567-1592, 2013.

\section{Author(s) Profile}

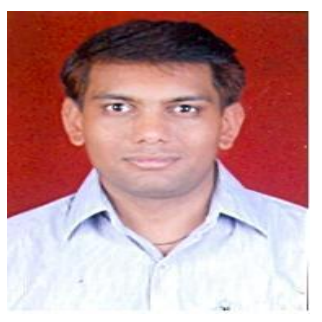

Mr. Manish Kumar Patidar received the B.E. degree in 2002 from R.G.P.V. Bhopal and M.E. degree in Digital Communication in 2005 from D.A.V.V. Indore. His area of specialization is Digital Communication, OFDM and Artificial neural network.

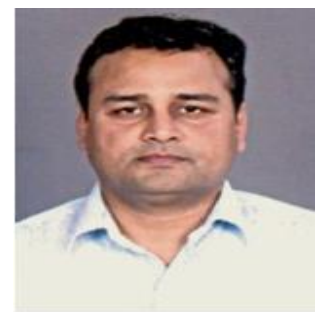

Dr. Amit Mishra received B.E. degree in Electronics Engineering from Marathwada University, Aurangabad, India in 1992 and the M.Tech. degree in the Engineering Systems and Ph.D. degree in Soft computing from the Dayalbagh Educational Institute (DEI), Agra, and Jamia Millia Islamia (Central University), Delhi, India in 2002 and 2010 respectively. His current interests include design of neural network architecture, OFDM wireless communication systems, nonlinear signal processing and optimization. 


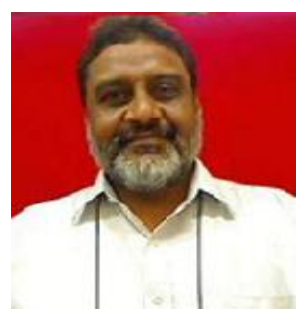

journals .

Prof. Rajiv Saxena received the degree of B. E., M. E. and Ph.D. in Electronics and Telecommunication engineering from Jabalpur University, Jiwaji University and Roorkee University, India respectively. He served as Professor/Principal/Head in different technical universities including Madhav Institute of Technology and Science (MITS), Gwalior, Thapar University, Patiala, Rustam Ji Institute of Technology, BSF Academy, Tekanpur etc. He executed two major research projects. He has supervised 15 $\mathrm{Ph} . \mathrm{D}$. degree candidates in the area of digital signal processing, and application of DSP tools in electronic systems. He has published about 70 research articles in refereed

How to cite this paper: Manish K. Patidar, A. Mishra, R. Saxena,"Probabilistic Constellation Extension Scheme for PAPR Reduction in OFDM Signals", IJWMT, vol.5, no.2, pp.12-21, 2015.DOI: 10.5815/ijwmt.2015.02.02 